\title{
Fossil groups of galaxies: Are they groups? Are they fossils?
}

\author{
Renato de Alencar Dupke ${ }^{1,2}$, Eric Miller ${ }^{3}$, Claudia Mendes de \\ Oliveira $^{4}$, Laerte Sodre $\mathbf{J r}^{4}$, Eli Rykoff ${ }^{5}$, Raimundo Lopes de Oliveira ${ }^{4}$ \\ and Rob Proctor ${ }^{4}$ \\ ${ }^{1}$ University of Michigan; ${ }^{2}$ ON/MCT $;{ }^{3}$ MIT Kavli Institute; ${ }^{4}$ IAG/USP $;{ }^{5}$ UCSB \\ email:rdupke@umich.edu
}

\begin{abstract}
Fossil groups present a puzzle to current theories of structure formation. Despite the low number of bright galaxies, their high velocity dispersions and high $T_{X}$ indicate cluster-like potential wells. Measured concentration parameters seem very high indicating early formation epochs in contradiction with the observed lack of large and well defined cooling cores. There are very few fossil groups with good quality X-ray data and their idiosyncrasies may enhance these apparent contradictions. The standard explanation for their formation suggests that bright galaxies within half the virial radii of these systems were wiped out by cannibalism forming the central galaxy. Since dry mergers, typically invoked to explain the formation of the central galaxies, are not expected to change the IGM energetics significantly, thus not preventing the formation of cooling cores, we investigate the scenario where recent gaseous (wet) mergers formed the central galaxy injecting energy and changing the chemistry of the IGM in fossil groups. We show a test for this scenario using fossil groups with enough X-ray flux in the Chandra X-ray Observatory archive by looking at individual metal abundance ratio distributions near the core. Secondary SN II powered winds would tend to erase the dominance of SN IA ejecta in the core of these systems and would help to erase previously existing cold cores. Strong SN II-powered galactic winds resulting from galaxy merging would be trapped by their deep potential wells reducing the central enhancement of SN Ia/SN II iron mass fraction ratio. The results indicate that there is a decrement in the ratio of SN Ia to SN II iron mass fraction in the central regions of the systems analyzed, varying from $99 \pm 1 \%$ in the outer regions to $85 \pm 2 \%$ within the cooling radius (Figure 1) and would inject enough energy into the IGM preventing central gas cooling. The results are consistent with a scenario of later formation epoch for fossil groups, as they are defined, when compared to galaxy clusters and normal groups.
\end{abstract}

Keywords. galaxies: clusters: general, X-rays: galaxies: clusters, surveys

Raimundo Lopes de Oliveira thanks FAPESP for the financial support.

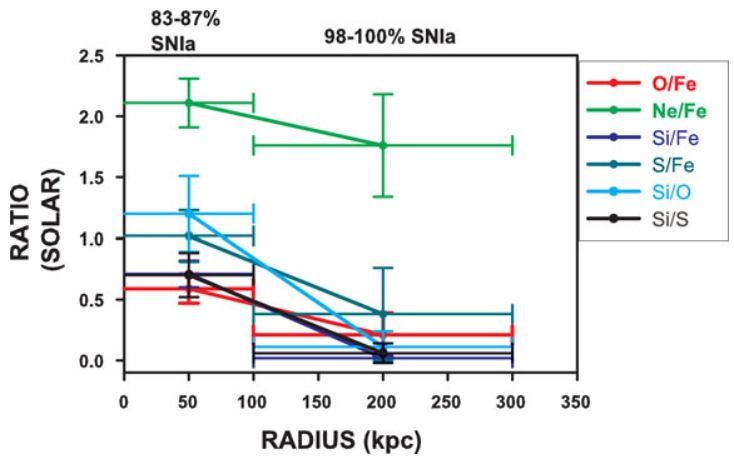

Figure 1. 